\title{
Microscopic kinetic analysis of space-charge induced optical microbunching in a relativistic electron beam
}

\author{
Agostino Marinelli ${ }^{1,2}$ and James B. Rosenzweig ${ }^{1}$ \\ ${ }^{1}$ Particle Beam Physics Laboratory, Department of Physics and Astronomy, \\ University of California Los Angeles, Los Angeles, California 90095, USA \\ ${ }^{2}$ Dipartimento SBAI, Università degli Studi di Roma La Sapienza, Via Antonio Scarpa 14, Rome, 00161, Italy
}

(Received 18 May 2010; published 29 November 2010)

\begin{abstract}
Longitudinal space-charge forces from density fluctuations generated by shot noise can be a major source of microbunching instability in relativistic high brightness electron beams. The gain in microbunching due to this effect is broadband, extending at least up to optical frequencies, where the induced structure on the beam distribution gives rise to effects such as coherent optical transition radiation. In the high-frequency regime, theoretical and computational analyses of microbunching formation require a full three-dimensional treatment. In this paper we address the problem of space-charge induced optical microbunching formation in the high-frequency limit when transverse thermal motion due to finite emittance is included for the first time. We derive an analytical description of this process based on the beam's plasma dielectric function. We discuss the effect of transverse temperature on the angular distribution of microbunching gain and its connection to the physics of Landau damping in longitudinal plasma oscillations. Application of the theory to a relevant experimental scenario is discussed. The analytical results obtained are then compared to the predictions arising from high resolution threedimensional molecular dynamics simulations.
\end{abstract}

DOI: $10.1103 /$ PhysRevSTAB.13.110703

PACS numbers: 29.20.Ej, 41.60.Cr, 41.75.Ht, 29.27.-a

\section{INTRODUCTION}

Density fluctuations due to shot noise in high brightness electron beams can couple to several impedance effects along accelerating systems, generating a broadband energy modulation. Longitudinal dispersion in bending magnets can transfer the modulation from energy to density, thus amplifying the microbunching well above the shot-noise level, an effect that is usually referred to as microbunching instability.

Microbunching instability is a critically important parasitic effect in high brightness relativistic electron sources, as it has the potential to seriously degrade the beam quality [1-7], thus compromising applications such as high gain free-electron lasers. Longitudinal space charge can be a source of microbunching growth at moderate energy $(<200 \mathrm{MeV})$ for wavelengths that are much shorter than the electron bunch length [7-10]. Indeed, it has been experimentally observed that space-charge induced microbunching instability may occur at quite short wavelengths, ranging down to the optical spectrum [11-13]. The highfrequency longitudinal space-charge interaction has also been proposed as a broadband amplifier to be used for novel seeding schemes in free-electron lasers [14].

In the high-frequency regime, the transverse beam size fulfills the condition $\sigma_{x} \gg \gamma \lambda / 2 \pi$, where $\sigma_{x}$ is the root mean square transverse size of the electron beam, $\lambda$ is the wavelength of interest, and the Lorentz factor $\gamma$ is the energy of the electron beam normalized to $m c^{2}$. In this limit, the Fourier components of the electric field generated by an uncorrelated electron distribution have a transverse correlation area which is much smaller than the electron beam section [15]. Under these conditions, the collective physics of the electron beam can be treated in analogy to an unbounded uniform plasma. Furthermore, in the highfrequency limit, the transverse dependence of the spacecharge fields strongly affects the electron dynamic [15] and the problem needs to be addressed with a fully threedimensional treatment. For this reason, we will refer to this limit as the three-dimensional limit. In this context, it is useful to define the bunching factor with an angular dependence [9]:

$$
b=\frac{1}{N} \sum_{n=1}^{N} e^{-i k\left[z_{n}+\sin \theta\left(x_{n} \cos \phi+y_{n} \sin \phi\right)\right],}
$$

where $N$ is the number of particles in the electron bunch, $\theta$ and $\phi$ are, respectively, the polar and azimuthal angles relative to the beam propagation axis $z, z_{n}$ is the longitudinal position along the bunch of the $n$th particle, and $x_{n}$ and $y_{n}$ are the transverse positions. With this definition, the bunching factor is effectively the three-dimensional Fourier transform of the electron density and accounts for any transverse structure in the microbunched beam.

A detailed computation of the angular distribution of the microbunching is of fundamental importance since the angular spectra of the radiation processes used to experimentally diagnose this effect, such as coherent optical transition radiation, are extremely sensitive to the transverse structure of the microbunched distribution [16,17]. With the 
assumption of transverse laminar motion, the microbunching geometry matches that of the longitudinal space-charge fields, yielding an angular width for the microbunching gain of $\theta_{c}=1 / \gamma$ [9]. Emittance effects in space-charge interactions, however, significantly modify the transverse structure of the microbunching pattern, and a kinetic treatment of the interaction between electrons and space-charge fields is needed to fully understand the physics of longitudinal space-charge-induced microbunching in this limit.

In this paper, we show that in the high-frequency limit, with the assumption that longitudinal motion is quasilaminar, the problem of space-charge interactions leading to microbunching growth becomes formally equivalent to that of one-dimensional plasma oscillations in a warm electron plasma. The theory of such electrostatic oscillations in thermal plasmas has been discussed in two seminal papers by Landau [18] and Jackson [19]; our present work represents a new application of these venerated techniques of mathematical physics in a radically different context than the one considered in the original papers. We show that transverse emittance induces strong Landau damping at high transverse spatial frequencies, significantly narrowing the angular width of the microbunching gain with respect to the characteristic angular width of the spacecharge fields. Finally, we compare the results of our analysis and those of high resolution molecular dynamics simulations, capable of investigating spatial features in the fields below the mean interparticle distance.

\section{LONGITUDINAL SPACE-CHARGE INDUCED MICROBUNCHING IN THE THREE-DIMENSIONAL LIMIT}

In this section we derive the equations that describe the formation of microbunching starting from shot noise through the effect of collective longitudinal space-charge forces. We base this analysis on the Vlasov equation, a kinetic approach that is appropriate for a warm plasma in which the number of beam electrons acting collectively to produce the electric and magnetic fields is much larger than unity. This is true when the condition $n_{0} \gamma^{2} \lambda^{3} \gg 1$ applies, where $n_{0}$ is the electron density and $\lambda$ is the wavelength of interest. This condition is equivalent to requiring that the wavelength of interest, as seen in the beam's rest frame $\gamma \lambda$, is much bigger than the rest frame mean interparticle distance $\left(\gamma / n_{0}\right)^{1 / 3}$. Obviously this approach precludes the possibility of describing beam crystallization processes, which require an analysis of particle to particle correlations on the scale of the mean interparticle distance in the beam's reference frame. Such analysis is the subject of current investigation and we will not discuss it here.

The Vlasov equation also ignores binary collisions, which is an excellent approximation for the time scales involved, as the mean-free beam-line distance for Coulomb scattering in a typical high brightness injector at moderate energy is of the order $100 \mathrm{~m} \mathrm{[20].}$
The Vlasov equation has been extensively used in literature for the analysis of shot-noise seeded instabilities. A notable example is the theory of self-amplified spontaneous emission in high gain free-electron lasers (see, for example, [21,22]). It could be argued that the Vlasov equation, being a deterministic evolution equation, is not suited for the description of shot noise, which requires a statistical treatment. However, while the Vlasov equation does not account for random interactions, namely particle to particle collisions, it can account for random perturbations in the initial particle distribution which, for every realization, evolve deterministically, which is the case for shot noise when collisional effects can be neglected.

It could also be argued that, since the Vlasov equation assumes a smooth distribution function, it cannot be used to describe shot-noise microbunching, which is generated by the intrinsic discreteness of the electron distribution. However, when we observe wavelengths that fulfill the condition $n_{0} \gamma^{2} \lambda^{3} \gg 1$, the discrete distribution function is integrated over many particles, generating random fluctuations in the collective fields which are not collisional, thus allowing the use of the Vlasov equation. Nevertheless, for a matter of convenience and ease of analysis, we will model the shot-noise spectrum as a discrete sum over the random positions of the individual electrons [see, for example, Eq. (16)]. The reader should not be confused by this and should keep in mind that our analysis only applies to the fraction of the spectrum that satisfies the condition $n_{0} \gamma^{2} \lambda^{3} \gg 1$.

To aid in analysis and to compare to previous theoretical work, we assume a coasting (nonaccelerating) beam with constant current. We also limit ourselves to the threedimensional limit of space-charge interactions $\left(\sigma_{x} \gg\right.$ $\gamma \lambda / 2 \pi)$. In the three-dimensional limit, edge effects due to the finite transverse size of the beam can be neglected and the 0th order charge density can be considered constant over the characteristic transverse scale of the problem.

Consistent with the current theoretical and experimental understanding of the process, we model the formation of microbunching as follows: the electron beam initially undergoes an external-force-free drift and space charge generates an energy modulation starting from shot noise. After the drift the electrons go through a series of optical elements which rearrange their longitudinal and transverse phase space coordinates according to a given transfer matrix $R_{i j}$ [23]. The electron beam particle distribution is described by a six-dimensional distribution function $f\left(\vec{x}_{\perp}, z, \vec{\beta}_{\perp}, p, \tau\right)$, where $\vec{x}_{\perp}$ is the transverse position, $z$ is the longitudinal position in the beam coordinate system, $\vec{\beta}_{\perp}$ is the transverse velocity normalized to the speed of light, $p=\Delta \gamma / \gamma$ is the relative energy deviation, and $\tau=c t$ where $c$ is the speed of light and $t$ is the time, measured from the beginning of the interaction. The distribution function is normalized to the total number of particles $N$. 
We expand the distribution function to first order in perturbation theory: $f=f_{0}+f_{1}$, with $\left|f_{1}\right| \ll\left|f_{0}\right|$. In the three-dimensional limit we can specify the following form for the 0th order distribution function: $f_{0}=$ $n_{0} f_{v}\left(\vec{\beta}_{\perp}, p\right)$, where $n_{0}$ is the local average particle density. For simplicity we assume that $f_{v}$ is isotropic in transverse velocity. Also, for simplicity, we will assume that $n_{0}$ is independent of time. This assumption is reasonable if the interaction happens close to a waist and the maximum distance from the waist is significantly shorter than the minimum $\beta$ function. If the waist is at the center of the drift the following condition has to apply: $L_{d} \lesssim \sigma_{x, 0} / \sigma_{\beta, 0}$, where $L_{d}$ is the length of the drift and $\sigma_{x, 0}$ and $\sigma_{\beta, 0}$ are, respectively, the root-mean-square (rms) transverse size of the beam and the transverse velocity spread (normalized to c) at the waist. If this condition does not apply, a possible solution is that of defining an equivalent drift length [9], shorter than the actual drift length, over which the beam density can be considered constant. In this paper we will not be concerned with this problem and we will assume that the condition $L_{d} \lesssim \sigma_{x, 0} / \sigma_{\beta, 0}$ is verified, leaving a detailed study of the effect of density variation for future investigation.

The collective beam dynamics in the drift are described by the Vlasov equation, coupled to the Maxwell equations. In the coasting beam case, we may derive the fields from the scalar potential computed in the beam rest frame. With these underlying assumptions the linearized Vlasov equation for the electrons in the drift reads

$$
\begin{gathered}
\frac{\partial f_{1}}{\partial \tau}+\vec{\beta}_{\perp} \cdot \nabla_{\perp} f_{1}+\frac{p}{\gamma^{2}} \frac{\partial f_{1}}{\partial z}+\frac{F_{z}}{\gamma m c^{2}} n_{0} \frac{\partial f_{v}}{\partial p} \\
+\frac{\vec{F}_{\perp}}{\gamma m c^{2}} \cdot n_{0} \frac{\partial f_{v}}{\partial \vec{\beta}}=0,
\end{gathered}
$$

where the approximation $\frac{d z}{d \tau} \approx p / \gamma^{2}$, valid for relativistic electrons, has been used. $F_{z}$ and $\vec{F}_{\perp}$ are, respectively, the longitudinal and transverse forces generated by the collective electric and magnetic fields of the electrons and can be computed solving Poisson's equation in the beam rest frame, where self-magnetic fields are negligible:

$$
\left[\nabla_{\perp}^{2}+\frac{1}{\gamma^{2}} \frac{\partial^{2}}{\partial z^{2}}\right] \phi=\frac{e}{\gamma \epsilon_{0}} \int f_{1} d p d^{2} \vec{\beta}
$$

Longitudinal forces are Lorentz invariant while transverse forces transform as $F_{\perp} \rightarrow F_{\perp} / \gamma$ going from the beam rest frame to the laboratory frame, we then have $F_{z}=\frac{e}{\gamma} \frac{\partial \phi}{\partial z}$ and $\vec{F}_{\perp}=\frac{e}{\gamma} \nabla_{\perp} \phi$.

It is convenient to solve Eqs. (2) and (3) in the LaplaceFourier domain. We give the following definitions:

$$
\hat{f}_{1}=\int f_{1} e^{-i\left(k_{z} z+\vec{k}_{\perp} \cdot \vec{x}\right)} d z d^{2} \vec{x}
$$

$$
\tilde{\hat{f}}_{1}=\int_{0}^{\infty} \hat{f}_{1} e^{-s \tau} d \tau
$$

and similarly for $F_{z}$ and $\vec{F}_{\perp}$. Since the system is isotropic in the transverse dimension we can set $\vec{k}_{\perp}=\hat{x} k_{x}$ without loss of generality. We also work in the paraxial approximation and set $k_{z}=k$ and $k_{x}=\theta k$. With the above definitions, the Laplace-Fourier transform of Eqs. (2) and (3) yields

$$
\begin{gathered}
\tilde{\hat{f}}_{1}-\left.\hat{f}_{1}\right|_{\tau=0}+i k\left(\theta \beta_{x}+\frac{p}{\gamma^{2}}\right) \tilde{\hat{f}}_{1} \\
+\frac{1}{\gamma m c^{2}} n_{0}\left(\tilde{\hat{F}}_{z} \frac{\partial f_{v}}{\partial p}+\tilde{\hat{F}}_{x} \frac{\partial f_{v}}{\partial \beta_{x}}\right)=0 \\
\tilde{\hat{F}}_{z}=-\frac{i}{k} \frac{e^{2}}{\epsilon_{0}} \frac{1}{1+(\gamma \theta)^{2}} \int \tilde{\hat{f}}_{1} d p d^{2} \vec{\beta} \\
\tilde{\hat{F}}_{x}=\theta \tilde{\hat{F}}_{z},
\end{gathered}
$$

where $\left.\hat{f}_{1}\right|_{\tau=0}$ is the spatial Fourier transform of the initial value of $f_{1}$.

From Eqs. (6) and (8) we can express $\tilde{\hat{f}}_{1}$ in terms of $\tilde{\hat{F}}_{z}$ :

$$
\begin{aligned}
\tilde{\hat{f}}_{1}= & \frac{1}{s+i k\left(\theta \beta_{x}+\frac{p}{\gamma^{2}}\right)} \\
& \times\left[\left.\hat{f}_{1}\right|_{\tau=0}-\frac{n_{0}}{\gamma m c^{2}} \tilde{\hat{F}}_{z}\left(\frac{\partial f_{v}}{\partial p}+\theta \frac{\partial f_{v}}{\partial \beta_{x}}\right)\right] .
\end{aligned}
$$

Inserting Eq. (9) into (6), recalling that $\tilde{\hat{F}}_{z}$ does not depend on $p$ and $\vec{\beta}$, we have

$$
\tilde{\hat{F}}_{z}=-\frac{i}{k} \frac{e^{2}}{\epsilon_{0} \epsilon_{p}} \frac{1}{1+(\gamma \theta)^{2}} \int \frac{\left.\hat{f}_{1}\right|_{\tau=0}}{s+i k\left(\theta \beta_{x}+\frac{p}{\gamma^{2}}\right)} d p d^{2} \vec{\beta}
$$

where $\epsilon_{p}$ is the beam's plasma dielectric function defined as

$$
\epsilon_{p}=1+\frac{\omega_{p}^{2}}{c^{2}\left[1+(\gamma \theta)^{2}\right]} \frac{\gamma^{2}}{i k} \int \frac{\frac{\partial f_{v}}{\partial p}+\theta \frac{\partial f_{v}}{\partial \beta_{x}}}{s+i k\left(\theta \beta_{x}+\frac{p}{\gamma^{2}}\right)} d p d^{2} \vec{\beta}
$$

with $\omega_{p}^{2}=\left(e^{2} n_{0}\right) /\left(\epsilon_{0} m \gamma^{3}\right)$ being the relativistic beam plasma frequency.

Inserting Eq. (10) back into Eq. (9), after some algebraic manipulation, we obtain the following expression for the first order distribution function: 


$$
\begin{aligned}
\tilde{\hat{f}}_{1}= & \frac{1}{s+i k\left(\theta \beta_{x}+\frac{p}{\gamma^{2}}\right)} \\
& \times\left[\left.\hat{f}_{1}\right|_{\tau=0}-\frac{1}{\epsilon_{p}} \frac{\omega_{p}^{2}}{c^{2}\left[1+(\gamma \theta)^{2}\right]}\left(\frac{\partial f_{v}}{\partial p}+\theta \frac{\partial f_{v}}{\partial \beta_{x}}\right) \frac{\gamma^{2}}{i k}\right. \\
& \left.\times \int \frac{\left.\hat{f}_{1}\right|_{\tau=0}}{s+i k\left(\theta \beta_{x}^{\prime}+\frac{p^{\prime}}{\gamma^{2}}\right)} d p^{\prime} d^{2} \vec{\beta}^{\prime}\right] .
\end{aligned}
$$

In what follows it will be understood that all the above integrals are analytically continued in the complex variable $s$ to the half-plane $\mathfrak{R}[s]<0$.

In performing the inverse Laplace transform we will only consider the zeros of the plasma dielectric function since these are the poles that describe the collective response of the electrons. Also, for the moment, we will only retain the $R_{56}$ matrix element in the transport matrix describing the optical elements after the drift. A more general treatment applicable to arbitrary linear phase space transformations is included in the Appendix.

The bunching factor is defined as the Fourier transform of the density perturbation normalized to the number of particles, i.e.,

$$
b\left(k_{z}, \vec{k}_{\perp}\right)=\frac{1}{N} \int f_{1} e^{-i\left(k_{z} z+\vec{k}_{\perp} \cdot \vec{x}\right)} d z d^{2} \vec{x} d p d^{2} \vec{\beta} .
$$

In order to arrive at the final prediction for the bunching factor, after the space-charge forces have modulated the particle energy distribution, we must account for the effect of longitudinal dispersion due to systems of bending elements (e.g. magnetic chicanes). The effect of the longitudinal dispersion, modeled through the $R_{56}$ matrix element, is that of shifting each particle's longitudinal position by an amount proportional to its energy deviation, i.e. $z \rightarrow z+$ $p R_{56}$. The effect of this transformation on the spatial Fourier components of the distribution function is that of introducing a phase shift proportional to the spatial rearrangement: $\hat{f}_{1} \rightarrow \hat{f}_{1} e^{-i k p R_{56}}$.

It follows that the bunching factor after the particles' rearrangement following the drift, can be expressed as

$$
\begin{aligned}
b= & -\frac{1}{N} \sum_{j} e^{s_{j} L_{d}} \frac{1}{\left.\frac{\partial \epsilon_{p}}{\partial s}\right|_{s=s_{j}}} \frac{\omega_{p}^{2}}{c^{2}\left[1+(\gamma \theta)^{2}\right]} \frac{\gamma^{2}}{i k} \\
& \times \int \frac{e^{-i k p R_{56}\left(\frac{\partial f_{v}}{\partial p}+\theta \frac{\partial f_{v}}{\partial \beta_{x}}\right)}}{s_{j}+i k\left(\theta \beta_{x}+\frac{p}{\gamma^{2}}\right)} d p d^{2} \vec{\beta} \\
& \times \int \frac{\left.\hat{f}_{1}\right|_{\tau=0}}{s_{j}+i k\left(\theta \beta_{x}+\frac{p}{\gamma^{2}}\right)} d p d^{2} \vec{\beta},
\end{aligned}
$$

where $L_{d}$ is the length of the drift and the sum is performed over all the zeros $s_{j}$ of the plasma dielectric function.

Integrating the first integral on the right-hand side of Eq. (14) by parts in $p$, and retaining only the term proportional to $R_{56}$, that accounts for the microbunching enhancement due to the longitudinal rearrangement, we obtain

$$
\begin{aligned}
b_{R_{56}}= & -\frac{1}{N} \sum_{j} e^{s_{j} L_{d}} \frac{1}{\left.\frac{\partial \epsilon_{p}}{\partial s}\right|_{s=s_{j}}} \frac{\omega_{p}^{2} R_{56} \gamma^{2}}{c^{2}\left[1+(\gamma \theta)^{2}\right]} \\
& \times \int \frac{e^{-i k p R_{56}} f_{v}}{s_{j}+i k\left(\theta \beta_{x}+\frac{p}{\gamma^{2}}\right)} d p d^{2} \vec{\beta} \\
& \times \int \frac{\left.\hat{f}_{1}\right|_{\tau=0}}{s_{j}+i k\left(\theta \beta_{x}+\frac{p}{\gamma^{2}}\right)} d p d^{2} \vec{\beta} .
\end{aligned}
$$

Finally, if the initial value of the perturbation $f_{1}$ results from shot noise, representing the individual particle positions in terms of $\delta$ functions, we may write the final integral in Eq. (15) as

$$
\begin{aligned}
& \int \frac{\left.\hat{f}_{1}\right|_{\tau=0}}{s+i k\left(\theta \beta_{x}+\frac{p}{\gamma^{2}}\right)} d p d^{2} \vec{\beta} \\
&=\left.\sum_{n=1}^{N} \frac{e^{-i\left(k z_{n}+k \theta x_{n}\right)}}{s+i k\left(\theta \beta_{x, n}+\frac{p_{n}}{\gamma^{2}}\right)}\right|_{\tau=0},
\end{aligned}
$$

where the particle positions are assumed random.

\section{THE LAMINAR AND QUASILAMINAR BEAM CASES}

Before proceeding to discuss the general case of interest in this paper, that of a transversely warm beam, we first examine analytically the limiting cases in which transverse thermal effects play a small role. To that end, in this section we derive a closed form expression for the microbunching in two simplified cases: the laminar and quasilaminar beam.

In the laminar beam approximation, where to lowest order particles are fixed in beam-frame position with respect to each other, we may write $f_{v}=\delta(p) \delta^{2}(\vec{\beta})$. In this case the plasma dielectric function can be easily computed analytically:

$$
\epsilon_{p}=1+\frac{\omega_{p}^{2}}{c^{2}} \frac{1}{s^{2}} .
$$

The zeros of $\epsilon_{p}$ are $s_{ \pm}= \pm i \omega_{p} / c$. Inserting $s_{ \pm}$in Eq. (15) we get

$$
\begin{aligned}
b_{R_{56}} & =-b_{0} \gamma^{2} R_{56} \frac{\omega_{p}}{c\left(1+[\gamma \theta]^{2}\right)} \sin \left(\frac{\omega_{p}}{c} L_{d}\right) \\
& \approx-b_{0}\left[\left(\frac{\gamma \omega_{p}}{c}\right)^{2} \frac{1}{1+(\gamma \theta)^{2}} R_{56} L_{d}\right],
\end{aligned}
$$

where $b_{0}=\left.\frac{1}{N} \sum_{n=1}^{N} e^{-i\left(k z_{n}+k \theta x_{n}\right)}\right|_{\tau=0}$, with $z_{n}$ and $x_{n}$ being randomly distributed, is the shot-noise bunching factor and we have made the approximation $\omega_{p} L_{d} / c \ll 1$.

To describe the quasilaminar beam approximation, we assume the following form of velocity distribution: $f_{v}=$ $\frac{1}{(2 \pi)^{3 / 2} \sigma_{\beta}^{2} \sigma_{p}} e^{-\left(p^{2} / 2 \sigma_{p}^{2}\right)-\left(\vec{\beta}^{2} / 2 \sigma_{\beta}^{2}\right)}$ with $k \sigma_{p} / \gamma^{2} \ll \omega_{p} / c$ and $k \theta \sigma_{\beta} \ll \omega_{p} / c$. These assumptions mean that the electron 
displacement due to thermal motion in a plasma period $\left(\tau_{p}=2 \pi / \omega_{p}\right)$ is much smaller than the wavelength $\lambda=$ $2 \pi / k$ longitudinally and $\lambda / \theta$ transversely. Since the plasma oscillation period sets the time scale for spacecharge effects, thermal effects become negligible when the thermal displacement in a plasma period is smaller than the length scale of the problem.

With this assumption, the plasma dielectric function is approximately equal to that found in the cold beam case and the bunching factor is given by

$$
b_{R_{56}} \approx-b_{0}\left[\left(\frac{\gamma \omega_{p}}{c}\right)^{2} \frac{1}{1+(\gamma \theta)^{2}} R_{56} L_{d}\right] e^{-\left[\left(k \sigma_{p} R_{56}\right)^{2} / 2\right]} .
$$

Finally, the microbunching gain is defined as the ratio of the statistical averages of the absolute values squared of the final to original bunching factor $g=\left\langle\left|b_{R_{56}}\right|^{2}\right\rangle /\left\langle\left|b_{0}\right|^{2}\right\rangle=$ $N\left\langle\left|b_{R_{56}}\right|^{2}\right\rangle$ and it is equal to

$$
g=\left[\left(\frac{\gamma \omega_{p}}{c}\right)^{2} \frac{1}{1+(\gamma \theta)^{2}} R_{56} L_{d}\right]^{2} e^{-\left(k \sigma_{p} R_{56}\right)^{2}} .
$$

In both the laminar and quasilaminar beam approximations, the electron thermal motion can be considered frozen on the scale of a plasma period. Thus, the microbunched distribution is transversely correlated on the same scale as the longitudinal Fourier components of the electric field generated by shot noise: $\gamma \lambda / 2 \pi$ (with $\lambda=2 \pi / k$ equal to the wavelength of interest). This results in a cutoff angle of $\theta_{c}=1 / \gamma$ in the microbunching gain.

Note that the same result (up to a geometric factor due to the assumptions on the 0th order charge density) has been obtained previously in [9].

\section{THE TRANSVERSELY WARM BEAM CASE}

In this section we treat the case that is most often found in experimental scenarios of interest: that of a beam that is transversely warm but longitudinally quasilaminar, i.e., we keep the assumption $k \sigma_{p} / \gamma^{2} \ll \omega_{p} / c$ but we make no assumptions on $\sigma_{\beta}$. Since space-charge forces naturally yield an angular cutoff of $1 / \gamma$, transverse temperature

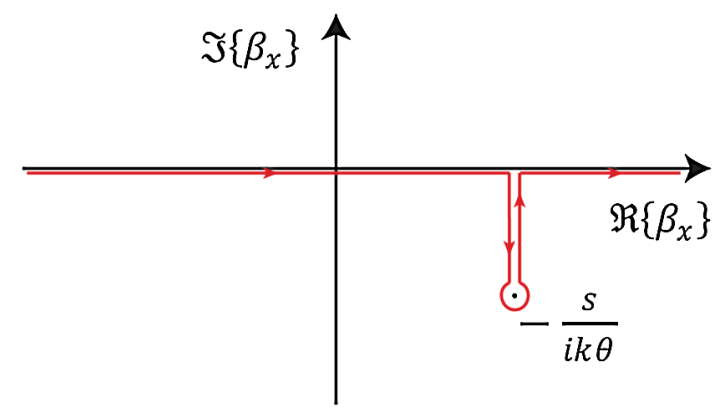

FIG. 1. Landau contour in the complex $\beta_{x}$ plane. The deformation of the integration path makes the plasma dielectric function analytical as a function of $s$. effects will be important only if $k \sigma_{\beta} \gtrsim \gamma \omega_{p} / c$, which is applicable for many relevant experimental situations.

By performing a double integration by parts (in $p$ and in $\left.\beta_{x}\right)$ in the term proportional to $\frac{\partial f_{v}}{\partial p}$ in (11), and performing the integration in $d p$ and $d \beta_{y}$, the plasma dielectric function can be expressed as

$$
\boldsymbol{\epsilon}_{p}=1+\frac{\omega_{p}^{2}}{c^{2}} \frac{1}{i k \theta} \int_{\tilde{c}} \frac{\frac{\partial f_{v}}{\partial \beta_{x}}}{s+i k\left(\theta \beta_{x}\right)} d \beta_{x} .
$$

In the case of a warm beam, the analyticity of $\epsilon_{p}$ as a function of the complex variable $s$ has to be enforced by deforming the integration path in (21) so that it runs in the complex plane below the singularity at $\beta_{x}=-s / i k \theta$ as shown in Fig. 1 (the resulting integration path is usually referred to as Landau contour [18] and we will denote it $\tilde{c}$ ).

The zeros of the plasma dielectric function, in this case, cannot be expressed in closed form. It is then useful to express Eq. (21) in dimensionless form. We give the following definitions: $k_{D}=\omega_{p} / c \sigma_{\beta}$ is the Debye wave number, which we employ to normalize the transverse wave number as $K=k \theta / k_{D}$; the Laplace variable $s$ is normalized to the plasma frequency as $\Omega=-c s / i \omega_{p}$; finally, we normalize the transverse velocity to the thermal velocity spread: $B=\beta_{x} / \sigma_{\beta}, F=\frac{1}{(2 \pi)^{1 / 2}} e^{-\left(B^{2} / 2\right)}$. Note that the Debye wavelength $\lambda_{D}=2 \pi / k_{D}$ is the transverse thermal displacement in a plasma period and is the fundamental parameter that describes thermal effects in warm plasmas. The resulting scaled beam plasma dielectric function is then

$$
\epsilon_{p}=1-\frac{1}{K^{2}} \int_{\tilde{c}} \frac{\frac{\partial F}{\partial B}}{B-\frac{\Omega}{K}} d B .
$$

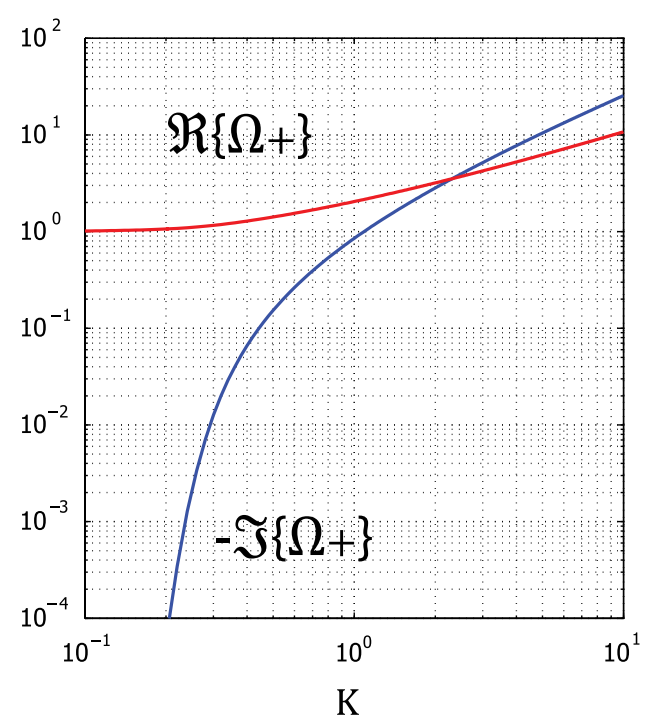

FIG. 2. Real part (red line) and imaginary part (blue line) of the roots of the plasma dielectric function for a transversely warm beam, as a function of the scaled wave number $K$ [19]. 
Note that the plasma dielectric function, as a function of $\Omega$, depends only on one external dimensionless parameter $K$. Since longitudinal thermal effects have been neglected and the system is azimuthally symmetric, this threedimensional problem reduces formally to that of onedimensional plasma oscillations discussed in [19].

The zeros $\Omega_{j}$ of (22) can be found numerically [19] and are, in general, complex. The imaginary part of the scaled frequency $\Omega_{j}$ is always negative, resulting in an exponential decay of the microbunching as a function of the drift length (this collisionless damping process due to thermal motion is usually denoted Landau damping). Also, if $\Omega_{R}-$ $i \Omega_{I}$ is a solution (with $\Omega_{R}, \Omega_{I}$ positive real numbers), then $-\Omega_{R}-i \Omega_{I}$ is also a solution [19]. We will thus denote as $\Omega_{ \pm}$the two dominant roots of the dielectric function (i.e. the roots with the smallest damping constant).

To develop an intuition on the physical processes involved, we examine Fig. 2 which shows the real and imaginary parts of the dominant root $\Omega_{+}$(see also [19]). The imaginary part of $-\Omega_{+}$is a growing function of $K$. For small values of $K$ the damping constant $-\mathfrak{s}\left\{\Omega_{+}\right\}$is small and can be neglected for drifts that are significantly shorter than a plasma wavelength, as is usual in most experimental situations. However, for $K>1$ the damping term is significantly bigger than 1 , resulting in a strong suppression of the microbunching gain at angles bigger than $k_{D} / k$. Equivalently, one could state that a transversely warm electron beam is unable to develop transverse structures on a scale that is smaller than $\lambda_{D}=2 \pi c \sigma_{\beta} / \omega_{p}$ under the effect of longitudinal space-charge forces.

To find the actual angular dependence of the microbunching gain we must compute the square of the absolute value of (15) and perform a statistical average. It can be shown that Eq. (15), with the above approximations, can be simplified to

$$
\begin{aligned}
b_{R_{56}}= & -i \frac{\omega_{p}}{c\left[1+(\gamma \theta)^{2}\right]} \gamma^{2} R_{56} e^{-\left[\left(k \sigma_{p} R_{56}\right)^{2} / 2\right]} \frac{1}{N} \\
& \times \sum_{j} e^{-i \Omega_{j}\left(\omega_{p} / c\right) L_{d}} \frac{K}{1-\frac{\Omega_{j}^{2}}{1+K^{2}}} \sum_{n=1}^{N} \frac{e^{-i\left(k z_{n}+k \theta x_{n}\right)}}{B_{n}-\frac{\Omega_{j}}{K}} .
\end{aligned}
$$

Taking the statistical average of the absolute value squared of (23), recalling that $\left\langle e^{-i k\left(z_{n}-z_{m}\right)-i k \theta\left(x_{n}-x_{m}\right)}\right\rangle=\delta_{n, m}$ (where $\delta_{n, m}$ is the Kronecker delta), from the definition of microbunching gain we obtain

$$
\begin{aligned}
g= & \left(\frac{\omega_{p}}{c\left[1+(\gamma \theta)^{2}\right]} \gamma^{2} R_{56}\right)^{2} e^{-\left(k \sigma_{p} R_{56}\right)^{2}} \int d B F(B) \\
& \times\left(\sum_{j} e^{-i \Omega_{j}\left(\omega_{p} / c\right) L_{d}} \frac{K}{1-\frac{\Omega_{j}^{2}}{1+K^{2}}} \frac{1}{B-\frac{\Omega_{j}}{K}}\right) \\
& \times\left(\sum_{j^{\prime}} e^{-i \Omega_{j^{\prime}}\left(\omega_{p} / c\right) L_{d}} \frac{K}{1-\frac{\Omega_{j^{\prime}}^{2}}{1+K^{2}}} \frac{1}{B-\frac{\Omega_{j^{\prime}}}{K}}\right)^{*} .
\end{aligned}
$$

In the dominant pole approximation, we keep only the two dominant roots in the summation in (24). In this case, expression (24) can be simplified to

$$
\begin{aligned}
g= & 2\left(\frac{\omega_{p}}{c\left[1+(\gamma \theta)^{2}\right]} \gamma^{2} R_{56}\right)^{2} e^{-\left(k \sigma_{p} R_{56}\right)^{2}}\left[\mid \frac{K e^{-i \Omega_{+}\left(\omega_{p} / c\right) L_{d}}}{1-\frac{\Omega_{+}^{2}}{1+K^{2}}}\right. \\
& \times\left.\right|^{2}\left(\left|\frac{K^{2}\left(1+K^{2}\right)}{\Omega_{+}^{2}}\right|-\sqrt{2 \pi} K \frac{\Re\left\{e^{-\left(\Omega_{+}^{2} / 2 K^{2}\right)}\right\}}{\Im\left\{\Omega_{+}\right\}}\right) \\
& -\Re\left\{( \frac { K e ^ { - i \Omega _ { + } ( \omega _ { p } / c ) L _ { d } } } { 1 - \frac { \Omega _ { + } ^ { 2 } } { 1 + K ^ { 2 } } } ) ^ { 2 } \left(\frac{K^{2}\left(1+K^{2}\right)}{\Omega_{+}^{2}}\right.\right. \\
& \left.\left.\left.-\sqrt{2 \pi} K \frac{e^{-\left(\Omega_{+}^{2} / 2 K^{2}\right)}}{\Omega_{+}}\right)\right\}\right] .
\end{aligned}
$$

Note that the quasilaminar beam case corresponds to $|K| \ll 1$. It can be shown that in this limit $\Omega_{+} \approx$ $1+\frac{3}{2} K^{2}-i \sqrt{\frac{\pi}{8}} e^{-\left(1+3 K^{2} / 2 K^{2}\right)} / K^{3}[18,19]$. With this asymptotic expression, Eq. (25) reduces to (20).

\section{NUMERICAL EXAMPLES}

As an example, we display a numerical evaluation of the angular dependence of the gain for the following beam parameters, corresponding to a typical electron beam produced by an rf photoinjector. We assume a uniform beam with a current of $I=40 \mathrm{~A}$ and an rms envelope size of $\sigma_{x}=85 \mu \mathrm{m}$ and an energy of $135 \mathrm{MeV}(\gamma=270)$. The length of the drift is $L_{d}=4 \mathrm{~m}$.

Figure 3 shows the angular dependence of the microbunching gain for several values of $\sigma_{\beta}$ for a wavelength of $\lambda=0.5 \mu \mathrm{m}$. We can see that for reasonable values of the emittance, transverse Landau damping can have an important role in the formation of microbunching, significantly

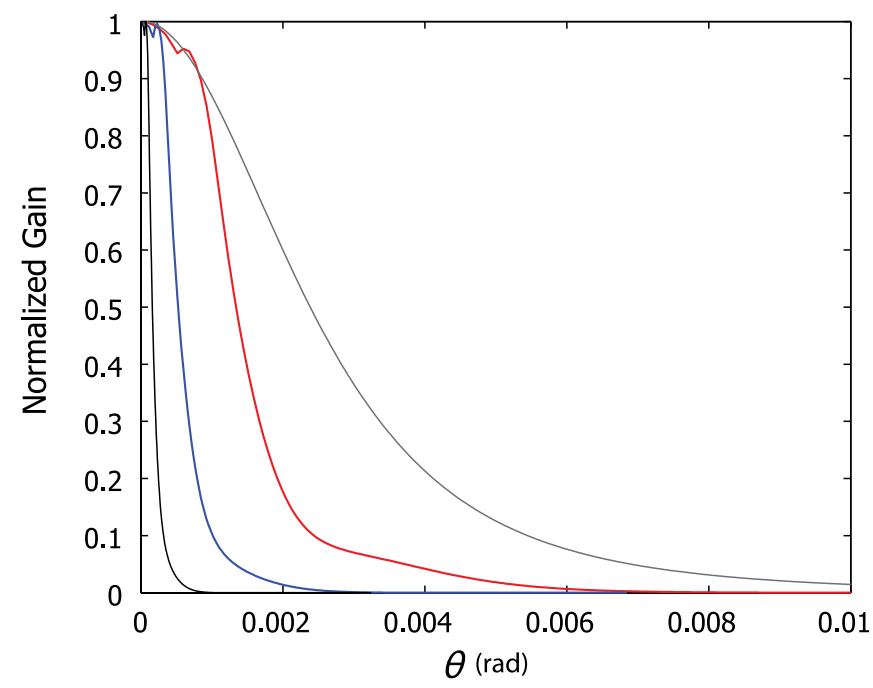

FIG. 3. Angular dependence of microbunching gain for different values of $\sigma_{\beta}$ corresponding to a normalized emittance of $\epsilon=0$ (gray line), $\epsilon=0.1 \mathrm{mmmrad}$ (red line), $\epsilon=0.5 \mathrm{~mm} \mathrm{mrad}$ (blue line), and $\epsilon=1 \mathrm{~mm} \mathrm{mrad}$ (black line). 
reducing the angular width of the gain with respect to the laminar beam case. Note that, for the $1 \mathrm{~mm}$ mrad case we have $L_{d} \approx 2 \sigma_{x, 0} / \sigma_{\beta, 0}$ and the results of the theory, in this case, are not accurate and should be interpreted with care.

\section{MOLECULAR DYNAMICS SIMULATIONS}

Numerical modeling of space-charge induced optical microbunching at optical and suboptical wavelengths is a challenging task since it requires high resolution and a great number of macroparticles. Ideally, to correctly reproduce shot-noise statistics in the electron distribution, each particle in the simulation should correspond to a particle in the beam. Also, resolution well below the optical spectrum is required to correctly compute the collective fields generated by the electrons.

Recently, the use of large computer clusters with parallel codes has allowed simulations with unprecedented resolution in the computation of self-fields, reaching down to the few $\mu \mathrm{m}$ level [24]. However, the resolution needed for optical and suboptical microbunching represents a serious challenge even for highly parallelized codes.

To model high-frequency space-charge phenomena with a low computational cost, we have created a code which computes the electron dynamics with periodic boundary conditions in all three dimensions. Periodicity allows one to limit the simulation window to a small fraction of the beam (few $\mu \mathrm{m}$ in the longitudinal dimension and several tens to few hundreds of $\mu \mathrm{m}$ transversely depending on the energy), thus reaching the resolution required (few $\mathrm{nm}$ to few tens of nm longitudinally). Also, due to the periodic boundary conditions, the code works in the threedimensional limit (see Fig. 4) since edge effects due to

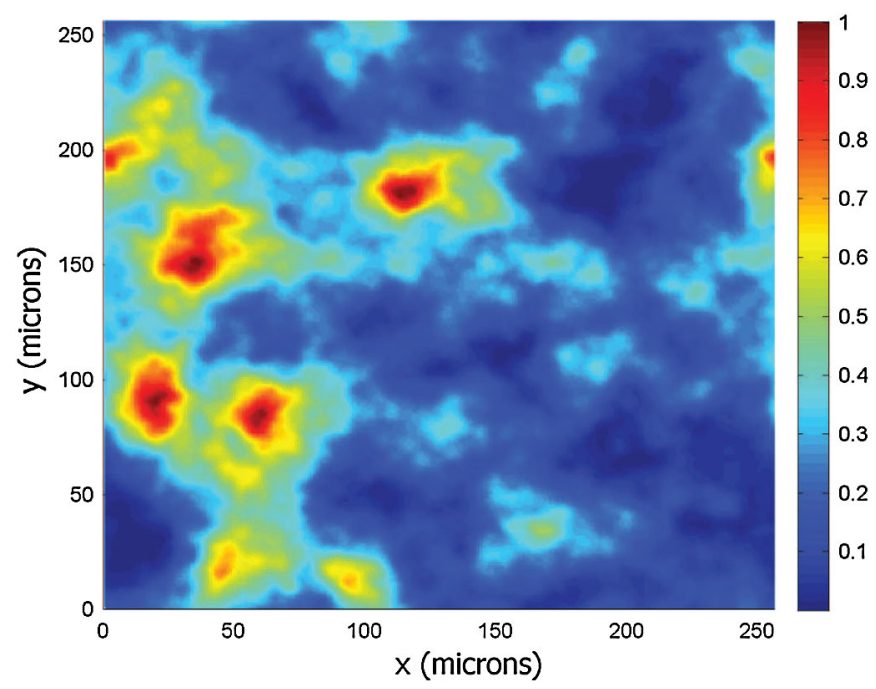

FIG. 4. Longitudinal Fourier transform of the electric field (in arbitrary units) generated by an uncorrelated electron distribution for $\gamma=270$ and $\lambda=0.5 \mu \mathrm{m}$, illustrating the features of the three-dimensional limit of space-charge fields. the finite size of the beam cannot be included. This limits the use of the code to very high-frequency phenomena.

The code has the capability of including external fields (accelerating and focusing) and internal fields. The highfrequency components of the collective fields are computed solving Poisson's equation in the beam rest frame with discrete Fourier transform methods.

We have performed simulations for the model assumed in Sec. II with no acceleration but only a free drift with subsequent rearrangement through longitudinal dispersion. The beam parameters are those described in Sec. V.

Figure 5 shows the $x-z$ trace space after longitudinal dispersion for two simulations with and without the effect of emittance. Note that in the zero emittance simulation, the position and size of the microbunches varies randomly
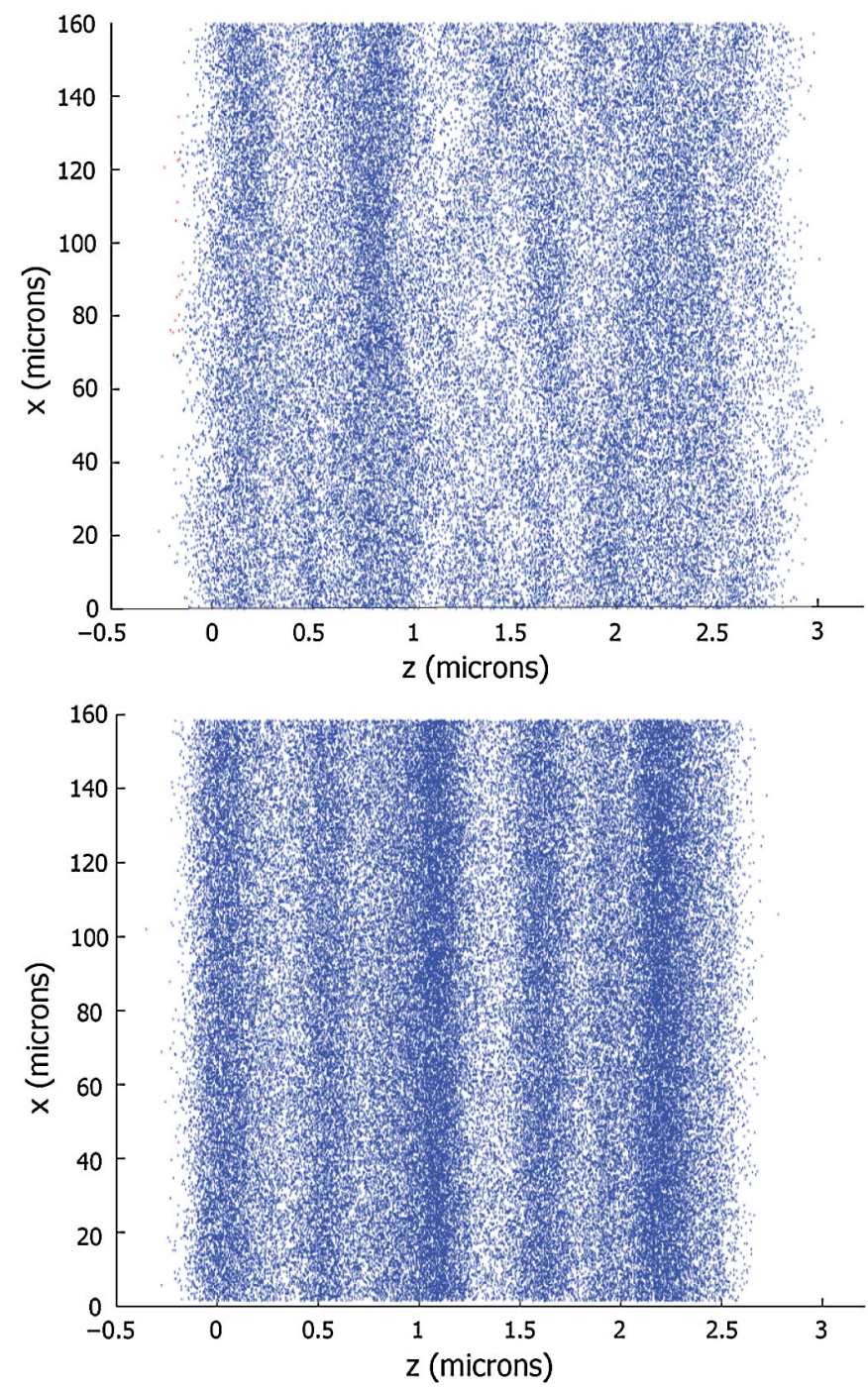

FIG. 5. $x-z$ trace space after a drift and longitudinal dispersion without emittance (upper plot) and with $1 \mathrm{~mm}$ mrad emittance (lower plot) showing the effect of Landau damping on the transverse structure of microbunching. 


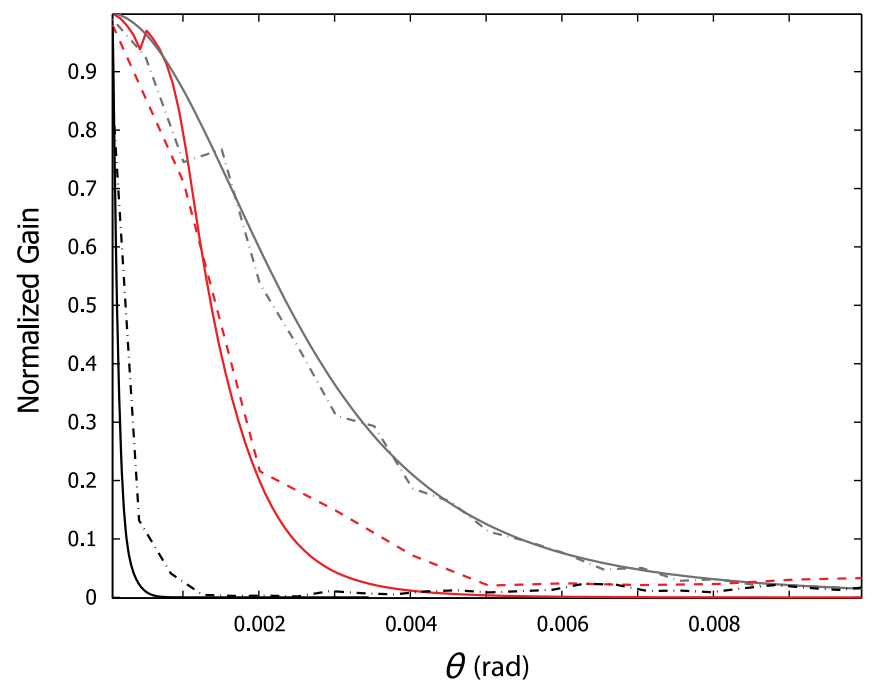

FIG. 6. Theoretical results (solid lines) versus numerical simulations (dashed lines) for different values of $\sigma_{\beta}$ corresponding to a normalized emittance of $\epsilon=0$ (gray line), $\epsilon=0.1 \mathrm{~mm} \mathrm{mrad}$ (red line), and $\epsilon=1 \mathrm{~mm}$ mrad (black line). The results of the simulations are averaged over 50 independent runs.

with the transverse position, resulting in a broad angular width of the microbunching pattern.

Figure 6 shows a comparison between the results of the theoretical model and the numerical simulations. The two are in good agreement, validating the theoretical analysis described earlier.

\section{CONCLUSIONS}

In this paper we discussed a kinetic analytical description of space-charge induced optical microbunching based on the beam plasma dielectric function.

The theory developed is fully three dimensional and accounts for the angular dependence of the microbunching in the three-dimensional limit. The kinetic approach allows for the inclusion of transverse thermal motion due to finite transverse emittance.

With the approximation that longitudinal motion is quasilaminar, which holds for typical high brightness electron beam parameters, the problem can be treated with the same mathematical methods used to describe one-dimensional plasma oscillations in thermal plasmas, as discussed in the work from Landau and Jackson [18,19]. In particular, the effect of Landau damping of transverse modes due to finite emittance has been discussed and found to be of great importance when typical values of emittance and beam plasma frequency are considered, significantly reducing the angular width of the microbunching gain with respect to the natural width $\theta_{c}=1 / \gamma$ of longitudinal space-charge fields.

With the further assumption of transverse quasilaminar motion, the results of our theory agree with those derived in previous papers on the same subject [9].
Finally, the results of our analysis have been compared to those generated by high resolution molecular dynamics simulations with periodic boundary conditions. The analytical and numerical results are found to be in good agreement, validating the theoretical analysis derived in this paper.

\section{ACKNOWLEDGMENTS}

The authors would like to acknowledge Professor G. J. Morales of UCLA for his invaluable advice on the subject of plasma oscillations. The authors are also extremely grateful to Dr. E. Hemsing of UCLA for many useful discussions and suggestions and to A. Pham for his help with numerical simulations. This work was supported by U.S. DOE Division of High Energy Physics under Contract No. DE-FG02-92ER40693, U.S. DOE Division of Basic Energy Sciences under Contract No. DE-FG0207ER46272, and Office of Naval Research Contract No. ONR N00014-06-1-0925.

\section{APPENDIX A: DERIVATION OF THE CLOSED FORM EXPRESSION FOR THE MICROBUNCHING GAIN}

With the assumption of longitudinal quasilaminar motion, Eq. (15) can be simplified taking advantage of the analytical properties of the plasma dispersion function, defined as $Z(\zeta)=\frac{1}{\sqrt{\pi}} \int_{\tilde{c}} d B \frac{e^{-B^{2}}}{B-\zeta}$. The plasma dispersion function is analytical in $\zeta$ in the whole complex plane and can be shown to have the following properties [19]:

$$
\begin{aligned}
& Z(\zeta)=e^{-\zeta^{2}} \operatorname{erfc}(-i \zeta) \\
& Z^{\prime}(\zeta)=-2-2 \zeta Z(\zeta),
\end{aligned}
$$

where $Z^{\prime}$ is the complex derivative of $Z$ and $\operatorname{erfc}(\zeta)$ is the complex valued complementary error function.

Integrating by part the plasma dielectric function, we obtain

$$
\epsilon_{p}=1-\frac{1}{2 K^{2}} Z^{\prime}\left(\frac{\Omega}{\sqrt{2} K}\right)
$$

Using Eqs. (A2) and (A3) we can express the plasma dielectric function and all its derivatives at the zeros of the plasma dielectric function. In particular we have

$$
\begin{gathered}
\left.Z\right|_{\epsilon_{p}=0}=-\frac{1+K^{2}}{\Omega} K \sqrt{2} \\
\left.Z^{\prime}\right|_{\epsilon_{p}=0}=2 K^{2} \\
\left.Z^{\prime \prime}\right|_{\epsilon_{p}=0}=2 \sqrt{2} K\left(\frac{1+K^{2}}{\Omega}-\Omega\right) .
\end{gathered}
$$


Finally we express the integrals in Eq. (15) as a function of $Z$ and its derivatives, with the approximation of longitudinal quasilaminarity:

$$
\int \frac{e^{-i k p R_{56}} f_{v}}{s_{j}+i k\left(\theta \beta_{x}+\frac{p}{\gamma^{2}}\right)} d p d^{2} \vec{\beta} \approx \frac{c e^{-\left[\left(k \sigma_{p} R_{56}\right)^{2} / 2\right]}}{i K \omega_{p} \sqrt{2}} Z\left(\frac{\Omega_{j}}{\sqrt{2} K}\right)
$$

$$
\left.\frac{\partial \epsilon_{p}}{\partial s}\right|_{s=s_{j}} \approx \frac{c}{i 2 \sqrt{2} K^{3} \omega_{p}} Z^{\prime \prime}\left(\frac{\Omega_{j}}{\sqrt{2} K}\right) .
$$

Equation (15) follows immediately substituting Eqs. (A4) and (A6) into Eqs. (A7) and (A8).

The same method can be applied to derive Eq. (25). By computing the partial fractions expansion of the integrand in Eq. (24) we can reduce it to a sum of $Z$ functions. Equation (25) then follows easily using the symmetry properties of $Z$ :

$$
\begin{aligned}
& Z(-\zeta)=-Z(\zeta)+2 i \sqrt{\pi} e^{-\zeta^{2}} \\
& Z\left(\zeta^{*}\right)=Z^{*}(\zeta)+2 i \sqrt{\pi} e^{-\zeta^{2}}
\end{aligned}
$$

\section{APPENDIX B: MICROBUNCHING GAIN WITH TRANSVERSE MATRIX ELEMENTS}

The model developed in the previous sections can be easily generalized to included transverse transport matrix elements. Note that the inclusion of Cartesian matrix elements breaks the azimuthal symmetry of the problem even if the transverse velocity distribution is isotropic. It is then necessary to set up the problem in a more general way. We adopt the following convention: $\vec{k}=k \hat{z}+$ $k \theta \cos (\phi) \hat{x}+k \theta \sin (\phi) \hat{y}$. We also define $\beta_{\|}$and $\beta_{\perp}$ the parallel and perpendicular components of transverse velocity with respect to $\vec{k}_{\perp}$. Finally we define $\tilde{k}_{x}=k\left[\theta \cos (\phi) R_{11}+R_{51}\right], \quad \tilde{k}_{y}=k\left[\theta \sin (\phi) R_{33}+R_{53}\right]$, $R_{x}=\theta \cos (\phi) R_{12}+R_{52}$, and $R_{y}=\theta \sin (\phi) R_{34}+R_{54}$. With these definitions, the derivation of the microbunching gain is similar to that of the previous sections with a few differences: $k \theta$ is now replaced by $\sqrt{\tilde{k}_{x}^{2}+\tilde{k}_{y}^{2}}$ and the integral $\int\left\{\left(e^{-i k p R_{56}} \frac{\partial f_{v}}{\partial p}\right) /\left[s_{j}+i k\left(\theta \beta_{x}+\frac{p}{\gamma^{2}}\right)\right]\right\} d p d^{2} \vec{\beta}$ is replaced by $\int\left\{\left(e^{-i k p R_{56}-i k R_{x} \beta_{x}-i k R_{y} \beta_{y}} \frac{\partial f_{v}}{\partial p}\right) /\left[s_{j}+i\left(\sqrt{\tilde{k}_{x}^{2}+\tilde{k}_{y}^{2}} \times\right.\right.\right.$ $\left.\left.\left.\beta_{\|}+\frac{p}{\gamma^{2}}\right)\right]\right\} d p d^{2} \vec{\beta}$. The final result is given by

$$
\begin{aligned}
g= & 2\left(\frac{\tilde{\omega}_{p}}{c\left(1+\gamma^{2}\left[\frac{\tilde{k}_{x}^{2}+\tilde{k}_{y}^{2}}{k^{2}}\right]\right)} \gamma^{2} R_{56}\right)^{2} e^{-\left(k \sigma_{p} R_{56}\right)^{2}-k^{2}\left(R_{x}^{2}+R_{y}^{2}\right) \sigma_{\beta}^{2}} \\
& \times\left[\left|\frac{K Z\left(\frac{\Omega_{+}}{\sqrt{2} K}+\frac{i k\left[R_{x} \cos (\phi)+R_{y} \sin (\phi)\right] \sigma_{\beta}}{\sqrt{2}}\right) e^{-i \Omega_{+}\left(\tilde{\omega}_{p} / c\right) L_{d}}}{\frac{\left(1+K^{2}\right)}{\Omega_{+}}-\Omega_{+}}\right|^{2}\right. \\
& \times\left(\left|\frac{K^{2}\left(1+K^{2}\right)}{\Omega_{+}^{2}}\right|-\sqrt{2 \pi} K \frac{\Re\left\{e^{-\left(\Omega^{2} / 2 K^{2}\right)}\right.}{\Im\{\Omega\}}\right) \\
& -\Re\left\{\left(\frac{K Z\left(\frac{\Omega_{+}}{\sqrt{2} K}+\frac{i k\left[R_{x} \cos (\phi)+R_{y} \sin (\phi)\right] \sigma_{\beta}}{\sqrt{2}}\right) e^{-i \Omega_{+}\left(\tilde{\omega}_{p} / c\right) L_{d}}}{\frac{\left(1+K^{2}\right)}{\Omega_{+}}-\Omega_{+}}\right)^{2}\right. \\
& \left.\left.\times\left(\frac{K^{2}\left(1+K^{2}\right)}{\Omega_{+}^{2}}-\sqrt{2 \pi} K \frac{e^{-\left(\Omega_{+}^{2} / 2 K^{2}\right)}}{\Omega_{+}}\right)\right\}\right]
\end{aligned}
$$

with the following substitution: $K=c \sigma_{\beta} \sqrt{\tilde{k}_{x}^{2}+\tilde{k}_{y}^{2}} / \omega_{p} . Z$ is the plasma dispersion function defined in Appendix A.

[1] M. Borland, Y.C. Chae, P. Emma, J. W. Lewellen, V. Bharadwaj, W. M. Fawley, P. Krejcik, C. Limborg, S. V. Milton, H. D. Nuhn, R. Soliday, and M. Woodley, Nucl. Instrum. Methods Phys. Res., Sect. A 483, 268 (2002).

[2] E. L. Saldin, E. A. Schneidmiller, and M. V. Yurkov, Nucl. Instrum. Methods Phys. Res., Sect. A 490, 1 (2002).

[3] S. Heifets, G. Stupakov, and S. Krinsky, Phys. Rev. ST Accel. Beams 5, 064401 (2002).

[4] Zhirong Huang and Kwang-Je Kim, Phys. Rev. ST Accel. Beams 5, 074401 (2002).

[5] Z. Huang, M. Borland, P. Emma, J. Wu, C. Limborg, G. Stupakov, and J. Welch, Phys. Rev. ST Accel. Beams 7, 074401 (2004).

[6] T. Shaftan and Z. Huang, Phys. Rev. ST Accel. Beams 7, 080702 (2004).

[7] Marco Venturini, Phys. Rev. ST Accel. Beams 10, 104401 (2007).

[8] E. L. Saldin, E. A. Schneidmiller, and M. V. Yurkov, in Proceedings of the 25th International Free Electron Laser Conference, and the 10th FEL Users Workshop [Nucl. Instrum. Methods Phys. Res., Sect. A 528, 355 (2004)].

[9] D. Ratner, A. Chao, and Z. Huang, in Proceedings of the 2008 Free-Electron Laser Conference (Jacow, Gyeongju, Korea, 2008), p. 338.

[10] A. Gover and E. Dyunin, Phys. Rev. Lett. 102, 154801 (2009).

[11] A. H. Lumpkin, N. S. Sereno, W. J. Berg, M. Borland, Y. Li, and S.J. Pasky, Phys. Rev. ST Accel. Beams 12, 080702 (2009).

[12] H. Loos et al., in Proceedings of the 2008 Free-Electron Laser Conference (Ref. [9]), p. 619.

[13] S. Wesch et al., in Proceedings of the 2009 Free-Electron Laser Conference (Jacow, Liverpool, UK, 2009), p. 485.

[14] E. A. Schneidmiller and M. V. Yurkov, arXiv:1003.5871.

[15] Marco Venturini, Phys. Rev. ST Accel. Beams 11, 034401 (2008).

[16] J. Rosenzweig, G. Travish, and A. Tremaine, Nucl. Instrum. Methods Phys. Res., Sect. A 365, 255 (1995). 
[17] A. Tremaine, J. B. Rosenzweig, S. Anderson, P. Frigola, M. Hogan, A. Murokh, C. Pellegrini, D. C. Nguyen, and R. L. Sheffield, Phys. Rev. Lett. 81, 5816 (1998).

[18] L. Landau, J. Phys. USSR 10, 25 (1946) [JETP 16, 574 (1946)].

[19] J. D. Jackson, J. Nucl. Energy, Part C 1, 171 (1960).

[20] A. Piwinski, DESY, Report No. 98-179, 1998.
[21] Kwang-Je Kim, Phys. Rev. Lett. 57, 1871 (1986).

[22] Ming Xie, Nucl. Instrum. Methods Phys. Res., Sect. A 475, 51 (2001).

[23] K. Brown, SLAC Report No. 75, 1982.

[24] J. Qiang, R. D. Ryne, M. Venturini, A. A. Zholents, and I. V. Pogorelov, Phys. Rev. ST Accel. Beams 12, 100702 (2009). 